University of Nebraska - Lincoln

DigitalCommons@University of Nebraska - Lincoln

October 1993

\title{
Triply differential (e,2e) cross sections for He from threshold to 50 eV above
}

C. Pan

University of Nebraska - Lincoln

Anthony F. Starace

University of Nebraska-Lincoln, astarace1@unl.edu

Follow this and additional works at: https://digitalcommons.unl.edu/physicsstarace

Part of the Physics Commons

Pan, C. and Starace, Anthony F., "Triply differential (e,2e) cross sections for He from threshold to $50 \mathrm{eV}$ above" (1993). Anthony F. Starace Publications. 128.

https://digitalcommons.unl.edu/physicsstarace/128

This Article is brought to you for free and open access by the Research Papers in Physics and Astronomy at DigitalCommons@University of Nebraska - Lincoln. It has been accepted for inclusion in Anthony F. Starace Publications by an authorized administrator of DigitalCommons@University of Nebraska - Lincoln. 


\title{
Triply differential (e,2e) cross sections for He from threshold to $50 \mathrm{eV}$ above
}

\author{
C. PAN and A.F. STARACE
}

Department of Physics and Astronomy, The University of Nebraska, Lincoln, NE 68588-0111, U.S.A.

\begin{abstract}
Distorted wave calculations of the triply differential cross sections for electron-impact ionization of $\mathrm{He}$ are presented for electrons sharing $\leq 50 \mathrm{eV}$ excess energy $\left(E_{\mathrm{x}}\right)$ and having the coplanar, $\theta_{12}=\pi$ geometry. The results are in good agreement with absolute experimental measurements of T. Rösel et al. [Phys. Rev. A 46 (1992) 2539-2552] and of A. J. Murray, M. B. Woolf, and F. H. Read [J.Phys. B 25 (1992) 3021-3036]. For final state electrons sharing $2 \mathrm{eV}$ excess energy equally, many-body perturbation theory corrections to the authors' first order (e,2e) triply differential cross sections [Phys. Rev. A 45 (1992) 4588-4603] are also presented. These results indicate that singlet-triplet interchannel coupling effects in the final state are very significant; that initial state correlations are significant near the perpendicular plane, i.e., $\theta_{1}=\theta_{2}=$ $\pi / 2$; and that the polarization effects we include are insignificant. Finally, comparison of our first order results with the semiclassical quantal theory results of D. S. F. Crothers [J. Phys. B 19 (1986) 463-483] shows excellent agreement for excess energies $E_{x} \geq 2 \mathrm{eV}$ and for $\theta_{1}=\theta_{2}=\pi / 2$; for $E_{x}<$ $2 \mathrm{eV}$, the two results diverge as $\mathrm{E}_{\mathrm{x}} \rightarrow 0$, indicating that experimental tests of the Wannier-PeterkopRau theory of three-body Coulomb breakup should be carried out in this energy region.
\end{abstract}

\section{INTRODUCTION}

Recently we have presented distorted-wave calculations of the triply differential cross sections for electron impact ionization of $\mathrm{H}, \mathrm{He}, \mathrm{Ne}, \mathrm{Ar}, \mathrm{Kr}, \mathrm{Xe}, \mathrm{Li}$, and $\mathrm{Mg}$ for final-state electrons departing in opposite directions $\left(\theta_{12}=\pi\right)$ and sharing between $0.5 \mathrm{eV}$ and $4 \mathrm{eV}$ excess energy $\left(E_{\mathrm{x}}\right)[1-4]$. The results for $\mathrm{H}$ and the rare gases were shown $[1,2]$ to agree well with the available relative experimental measurements [5-8]. In particular, they provided an interpretation of the observed target dependence of the electron angular distributions for $\mathrm{H}$ and $\mathrm{He}$ targets [6] in terms of short-range effects on the $s$-wave phase shifts of both incident and final-state continuum electrons [1-2]. As compared to the theoretical $(\mathrm{e}, 2 \mathrm{e})$ calculations for $\mathrm{H}$ and $\mathrm{He}$ at these near-threshold energies [9-11], our results [1-4] showed the importance of distortion, of full treatment of non-local exchange interactions, of inclusion of both singlet and triplet partial waves, and of mutual screening interactions employing effective charges which satisfy proper asymptotic boundary conditions [12-14]. Furthermore, large differences in the absolute values of the triply differential cross sections predicted by different theoretical calculations $[1,2,9,10]$ were found [15]. Recent absolute experimental measurements for electron-impact ionization of $\mathrm{He}$ at $2 \mathrm{eV}$ and $4 \mathrm{eV}$ excess energy, however, have confirmed our values for the absolute triply differential cross sections $[16,17]$.

Stimulated in large part by the availability of absolute experimental measurements, we present in this paper results of three further theoretical studies of the triply differential cross section for the $(e, 2 e)$ process in He for final state electrons in the $\theta_{12}=\pi$ geometry. The first study concerns extension of our first order, distorted partial wave procedure $[1-4]$ to the intermediate energy region, up to $E_{x}=50 \mathrm{eV}$. The results obtained for the triply differential cross sections in the perpendicular plane, $\theta_{1}=\theta_{2}=\pi / 2$, are compared with the absolute measurements of Murray, Woolf, and Read [18] as well as with those of Rösel et al. [16,17]. 

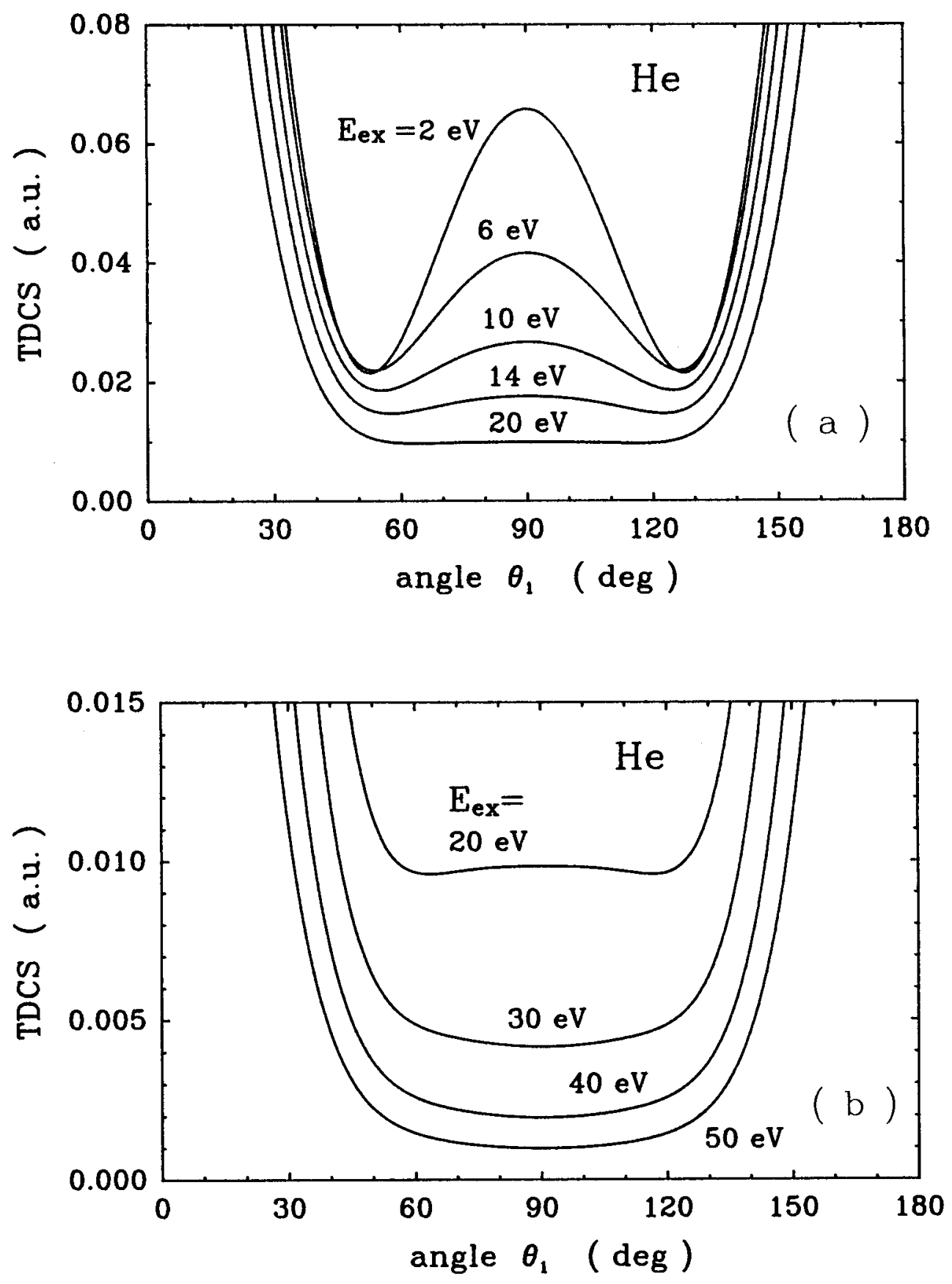

Figure 1. Triply differential (e,2e) cross sections for $\mathrm{Hc}$ for final states having $\theta_{12}=\pi$ and the continuum electrons sharing the excess energy $E_{X}$ equally. (a) $E_{x}=2 \mathrm{eV}, 6 \mathrm{eV}, 10 \mathrm{eV}, 14 \mathrm{eV}$, and $20 \mathrm{eV}$; (b) $E_{X}=20 \mathrm{eV}, 30 \mathrm{eV}, 40 \mathrm{eV}$, and $50 \mathrm{eV}$. 
The second study concerns the influence of electron correlation effects for the case $E_{x}=2 \mathrm{eV}$. We use many-body perturbation theory to calculate these effects and we compare our results with the absolute measurements of Rösel et al. [16]. In a third study, we compare our results with theoretical results of Crothers [11(a)]. This comparison enables us to indicate to experimentalists the appropriate energy range for testing the Wannier-Peterkop-Rau [19-21] theory for breakup of three-body Coulomb systems.

\section{INTERMEDIATE ENERGY RESULTS}

In Fig. 1 we present our triply differential $(e, 2 e)$ cross sections for continuum electrons sharing the excess energy equally. Over the range $2 \mathrm{eV} \leq \mathrm{E}_{\mathrm{x}} \leq 20 \mathrm{eV}$ one sees clearly in Fig. 1(a) that the cross section maximum in the perpendicular plane $\left(\theta_{1}=\theta_{2}=\pi / 2\right)$ decreases rapidly with increasing $E_{x}$ and has nearly disappeared by $E_{x}=20 \mathrm{eV}$. In Fig. 1(b) one sees that on a finer energy scale, there is actually still a local maximum at $\theta_{1}=90^{\circ}$ for $E=20 \mathrm{eV}$, but that this maximum vanishes for $30 \mathrm{eV} \leq \mathrm{E}_{\mathrm{x}} \leq 50 \mathrm{eV}$. We have limited the maximum value of $E_{x}$ to $50 \mathrm{eV}$ in order to avoid the energy region in which doubly-excited resonance structures may influence the cross sections. For the equal energy sharing case considered here, this will occur when $E_{x} / 2+I_{H e}$ equals the energy of the lowest doubly excited state, where $I_{H e}=24.6 \mathrm{eV}$. Since the doubly excited states of He are located above about $60 \mathrm{eV}$, our maximum value of $\mathrm{E}_{\mathrm{X}}=50 \mathrm{eV}$ is thus $20 \mathrm{eV}$ below the doubly excited energy region.

In Fig. 2 we present triply differential (e,2e) cross sections for the case of unequal energy sharing in which $\varepsilon_{1}=E_{x}-5 \mathrm{eV}$ and $\varepsilon_{2}=5 \mathrm{eV}$. One sees that the cross sections are asymmetric about $\theta_{1}=\theta_{2}=\pi / 2$ and that up to the highest excess energy there is a cross section maximum in the region $60^{\circ} \lesssim \theta_{1} \lesssim 90^{\circ}$, although it is becoming less prominent with increasing $E_{\mathrm{x}}$. In these calculations, we have chosen the maximum value of $\mathrm{E}_{\mathrm{x}}(40 \mathrm{eV})$ to be just below that at which doubly-excited state structures may appear, i.e., when $\left(\mathrm{E}_{\mathrm{x}}-5 \mathrm{eV}\right.$ $\left.+\mathrm{I}_{\mathrm{He}}\right) \approx 60 \mathrm{eV}$.

In Fig. 3 we compare our results for the equal energy sharing case (cf. Fig. 1) with intermediate energy absolute experimental results obtained in perpendicular plane measurements by Murray, Woolf, and Read [18]. For $\theta_{1}=\theta_{2}=90^{\circ}$, our triply differential cross sections are in the plane perpendicular to the incident electron beam and may be compared with such experimental measurements. Our results are plotted over the energy range $1 \mathrm{eV} \leq \mathrm{E}_{\mathrm{x}} \leq 90 \mathrm{eV}$ and the cross sections are plotted on a logarithmic scale because of the rapid decrease of the equal energy sharing cross section in the perpendicular plane. Near threshold we compare also with the absolute experimental measurements of Rösel et al. $[16,17]$ for $\mathrm{E}_{\mathrm{x}}=2 \mathrm{eV}$ and $4 \mathrm{eV}$.

In Fig. 3 our theoretical results are extended into the region of doubly excited state resonances above $\mathrm{E}_{\mathrm{x}}=$ $70 \mathrm{eV}$. Such resonance effects are not included in our calculations, but may influence the experimental values in this energy region. We have nevertheless presented our results in this region in order to make comparison with the absolute measurement of Gélébart and Tweed [21] at $\mathrm{E}_{\mathrm{x}}=\left(100 \mathrm{eV}-\mathrm{I}_{\mathrm{He}}\right) \approx 75.4 \mathrm{eV}$. The (relative) experimental measurements of Murray, Woolf, and Read [18] have been put on an absolute basis in part by normalization to the absolute measurements of Ref. [21]. These latter results, however, have recently been found to require a reduction by 18 percent [22]. Such a reduction may lead to improved agreement of our results with those of Refs. [18] and [21]. Even without this correction, on the logarithmic scale of Fig. 3 one sees that our results on the whole agree quite well with the absolute experimental measurements. This good agreement indicates the usefulness of a partial wave treatment for $(e, 2 e)$ triply differential cross sections in the intermediate energy region.

\section{ELECTRON CORRELATION EFFECTS}

In Fig. 4 we compare theoretical and experimental results for the triply differential $(e, 2 e)$ cross section for $\mathrm{He}$ for the $\theta_{12}=\pi$ geometry and for equal energy sharing, with $\varepsilon_{1}=\varepsilon_{2}=1 \mathrm{eV}$. Two sets of experimental data are shown: the relative measurements of Selles, Huetz, and Mazeau [5] and the absolute measurements of Rösel et al. $[16,17]$. The former [5] have been normalized to the latter $[16,17]$ at $\theta_{1}=50^{\circ}$. The four theoretical curves include our first order, distorted wave results (cf. the long-dashed curve in Fig. 4) as well as results including successively three kinds of electron correlation effects. These effects have been taken into account using many-body perturbation theory, as discussed in detail elsewhere [4]. We discuss the influence of each of these three electron correlation effects in turn. 


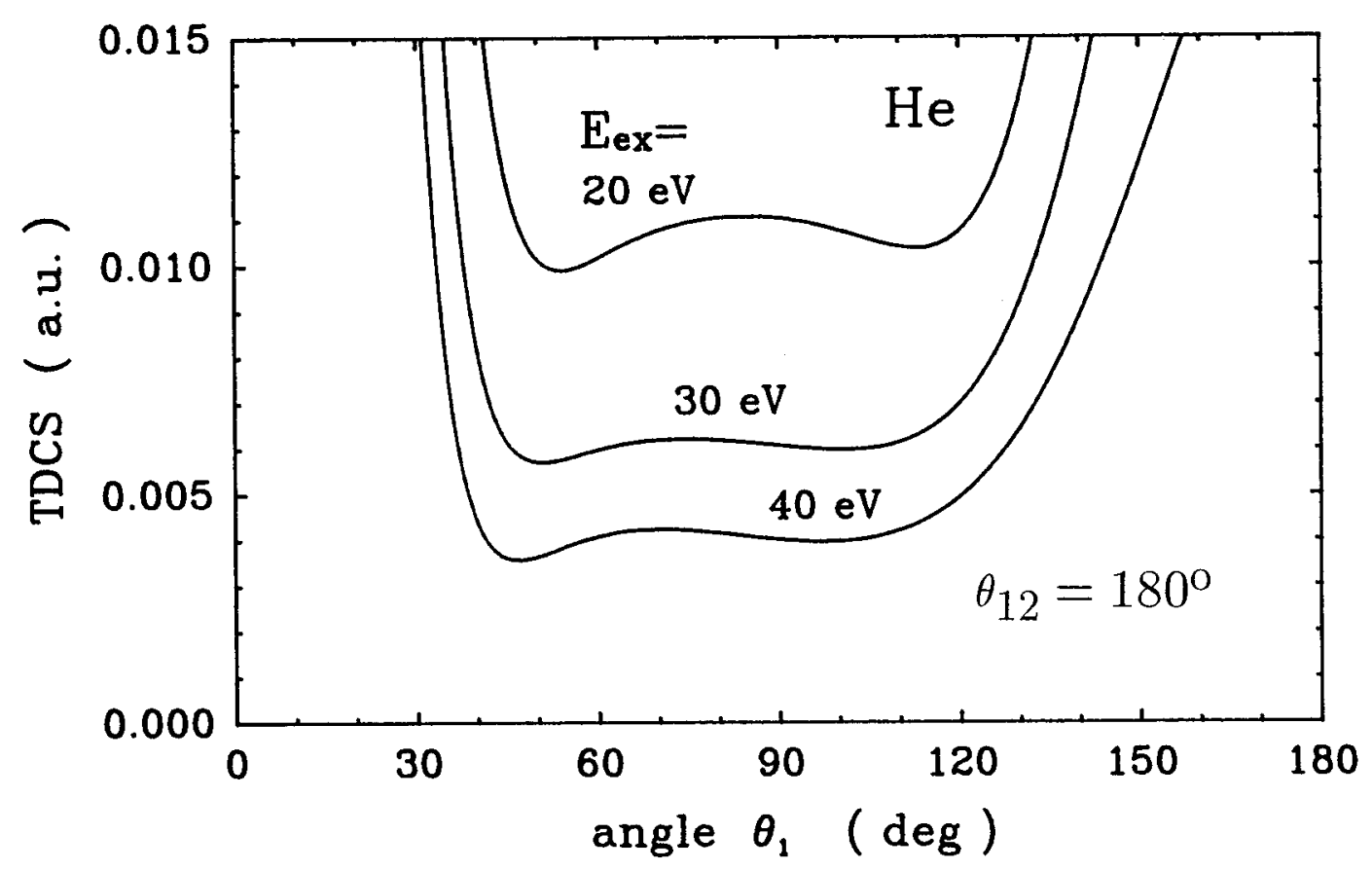

Figure 2. Triply differential (e,2e) cross sections for He for final states having $\theta_{12}=\pi$ and the continuum electrons having unequal energy-sharing with $\varepsilon_{1}=\mathrm{E}_{\mathrm{X}}-5 \mathrm{eV}$ and $\varepsilon_{2}=5 \mathrm{eV}$. Results are shown for $\mathrm{E}_{\mathrm{X}}=20 \mathrm{eV}, 30 \mathrm{eV}$, and $40 \mathrm{eV}$, or $\varepsilon_{1} / \varepsilon_{2}=$ 4,6 , and 8 respectively.

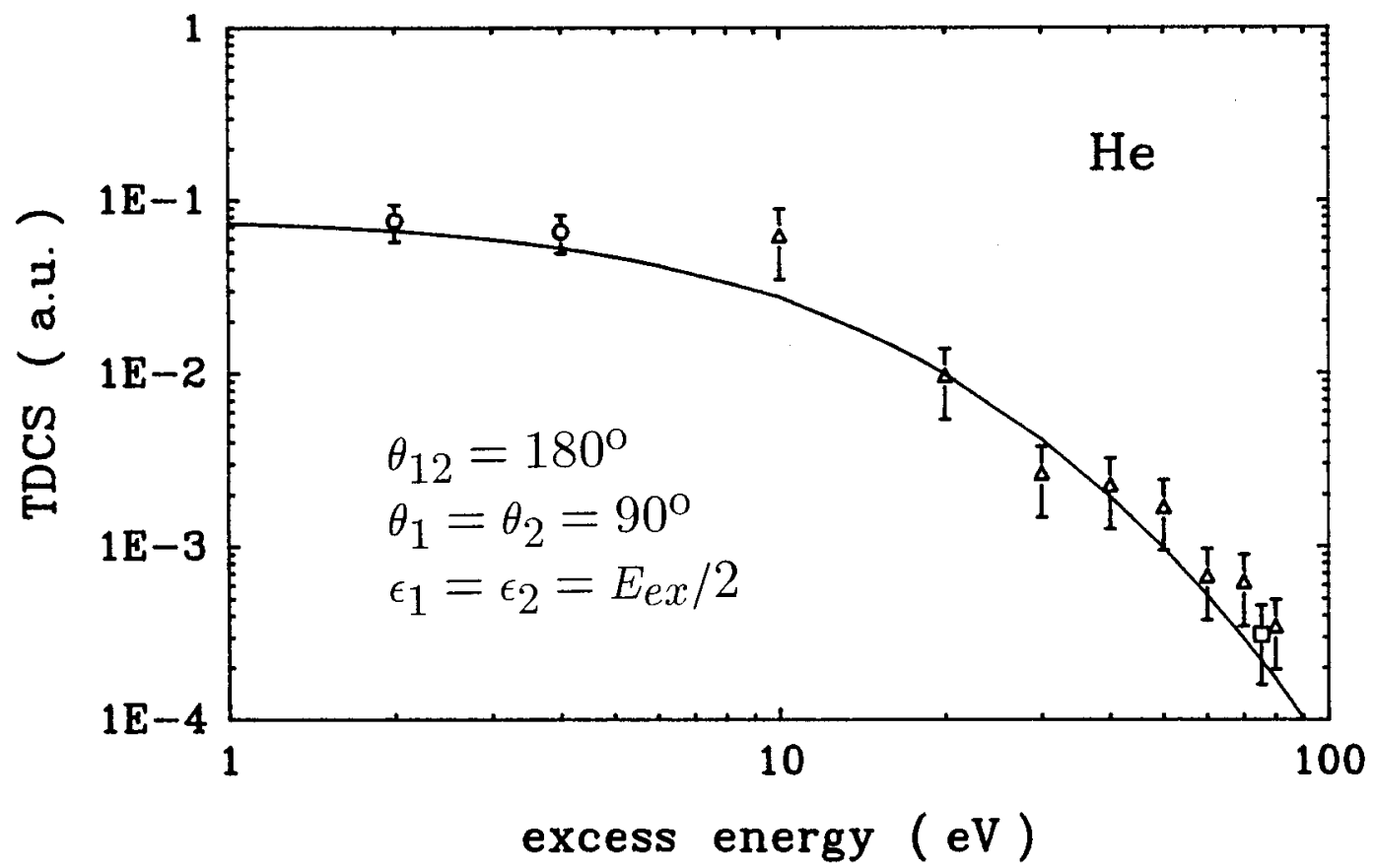

Figure 3. Triply differential (e,2e) cross sections for He for final states having $\theta_{12}=\pi, \theta_{1}=\theta_{2}=\pi / 2$, and equal energy sharing plotted vs. $\mathrm{E}_{\mathrm{X}}$. Solid curve: present distorted wave theoretical results. Open circles: absolute experimental results of Rosel et al., Ref. [16,17]. Open triangles: absolute experimental results of Murray, Woolf, and Read, Ref. [18]. Open squares: absolute experimental results of Gélébart and Tweed, Ref. [21]. 
The dotted curve in Fig. 4 includes the effect of final-state interchannel coupling. Because the $\mathrm{e}^{-}-\mathrm{He}$ system is a three-electron system, the spin of the pair of continuum electrons in the final state is not a good quantum number. This contrasts with the case of $\mathrm{e}^{-}-\mathrm{H}$ scattering (ignoring, of course, the nuclear spin). The spin $1 / 2$ electron in the $\mathrm{He}^{+}$core thus couples singlet and triplet final-state partial waves of the pair of continuum electrons. These interchannel electron correlation effects were taken into account by solving close-coupling-type equations in the final state $[2,4]$. The results shown in Fig. 4 indicate that this effect worsens agreement between theory and experiment in the perpendicular plane (i.e., $\theta_{1}=90^{\circ}$ ). However, it gives a dramatically improved agreement with experiment at both small and large angles (i.e., for $\theta_{1} \leq 50^{\circ}$ and $\theta_{1} \geq 130^{\circ}$ ).

The short-dashed curve in Fig. 4 includes, in addition to interchannel effects, the effect of certain initial state electron polarization interactions. Many-body perturbation theory diagrams for these effects have been given in Ref. [4]. These interactions simply describe the fact that the incident electron may induce virtual excitations of one of the ground state electrons in He. [Note that we are very far from the energy region of the doubly excited states of $\mathrm{He}$ and have therefore calculated only the real part of these interactions and ignored the imaginary part.] As shown in Fig. 4, in the perpendicular plane (i.e., $\theta_{1}=90^{\circ}$ ) such effects are so small that the dotted curve is indistinguishable from the short-dashed curve. In the "wings" of the triply differential cross section (i.e., for $\theta_{1} \leq 50^{\circ}$ and $\theta_{1} \geq 130^{\circ}$ ) the dotted curve is slightly higher than the short-dashed curve, giving slightly improved agreement with experiment.

The solid curve in Fig. 4 includes not only the two electron correlation effects already discussed but also the effect of ground-state correlations in He. These ground-state correlation effects account for the fact that the independent particle model description of He as a $1 \mathrm{~s}^{2}$, closed-shell atom is inadequate. Rather, both of the two electrons in He may also be virtually excited part of the time. The triply differential cross section is affected whenever the incident electron interacts not with a pair of 1s-electrons but with a pair of virtually excited electrons. Fig. 4 shows that this effect is strongest in the perpendicular plane (i.e., for $\theta_{1}=90^{\circ}$ ), giving a cross section that now is reduced below the error bars of the absolute experimental measurements. In the "wings" of the triply differential cross section, however (i.e., for $\theta_{1} \leq 50^{\circ}$ and $\theta_{1} \geq 130^{\circ}$ ), the solid curve is barely changed from the results shown by the dotted curve and by the short-dashed curve. Indeed, the solid curve lies between these two other results and is thus still in excellent agreement with experiment.

We conclude, therefore, that final-state interchannel interactions are very important for improving agreement between theory and experiment in the "wings" of the triply differential cross section (i.e., for $\theta_{1} \leq 50^{\circ}$ and $\theta_{1} \geq 130^{\circ}$ ). Polarization effects induced by the incident electron appear to be negligible for He. Finally, both interchannel interactions and ground state correlation effects worsen the agreement between theory and experiment in the perpendicular plane (i.e., for $\theta_{1}=90^{\circ}$ ), making this region an interesting one for future experimental and theoretical investigation.

\section{WANNIER-PETERKOP-RAU THRESHOLD LAW}

As is shown in Fig. 10 of Ref. [17], the calculations by Crothers [11(a)], by Pan and Starace [2], and the latest results of Jones and Madison [17] (which include a local approximation for the exchange interaction) all agree well with the absolute experimental measurements of Rösel et al. [17] for the triply differential cross section of $\mathrm{He}$ in the $\theta_{12}=\pi$ geometry and for $\theta_{1}=90^{\circ}$ and $E_{x}=2 \mathrm{eV}$. However, the energy behaviors of the triply differential cross sections (TDCS) for $\theta_{12}=\pi$ are very different near threshold and these differences may be exploited in order to test the Wannier-Peterkop-Rau (WPR) theory of breakup processes involving interacting three-body Coulomb systems [19-21].

As discussed by Peterkop [20(b)] and by Selles, Huetz, and Mazeau [5], the TDCS for He for the $\theta_{12}=\pi$ geometry has an energy dependence of $\left(E_{x}\right)^{1.127-1.5}=\left(E_{x}\right)^{-0.373}$ in the WPR theory. This diverging behavior of the TDCS as $E_{x} \rightarrow 0$ is related to the predicted rapid narrowing of the width of the distribution with respect to $\theta_{12}$ in the region of $\theta_{12}=\pi$, which varies as $E_{x}^{0.25}$. Crothers' calculations [11(a)] are consistent with this WPR prediction.

On the other hand, the TDCS's for the $\theta_{12}=\pi$ case given by calculations using the effective-charge approximation $[2,10,12-14,17]$ are independent of the excess energy in the $E_{x} \rightarrow 0$ limit. This threshold behavior can be verified by analyzing the wave functions used in such calculations. Thus, the agreement of 


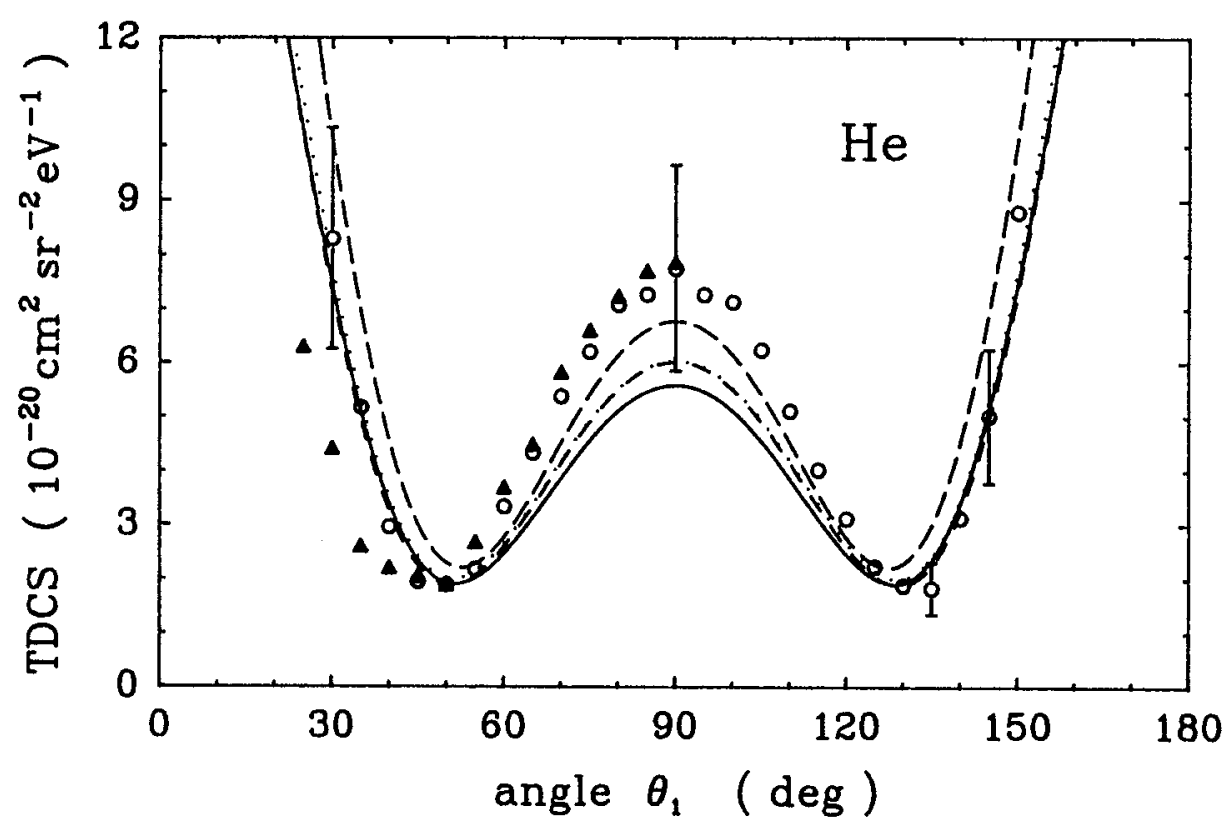

Figure 4. Triply differential (e,2e) cross sections for a He-atom target for final states having $\theta_{12}=\pi$ and the continuum electrons sharing $2 \mathrm{eV}$ excess energy equally. Open circles: absolute experimental results of Rosel et al., Ref. [16]. Solid triangles: experimental results of Selles, Huetz and Mazeau, Ref. [5], fitted to the result of Rösel $e t$ al . at $\theta_{1}=50^{\circ}$. Curves are the present theoretical results. Long-dashed curve: first-order results. Dotted curve: results also including the final-state interchannel interactions between singlet and triplet states. Short-dashed curve: results also including certain $\mathrm{e}^{-}-\mathrm{He}$ polarization interactions. Solid curve: results also including He ground state correlations.

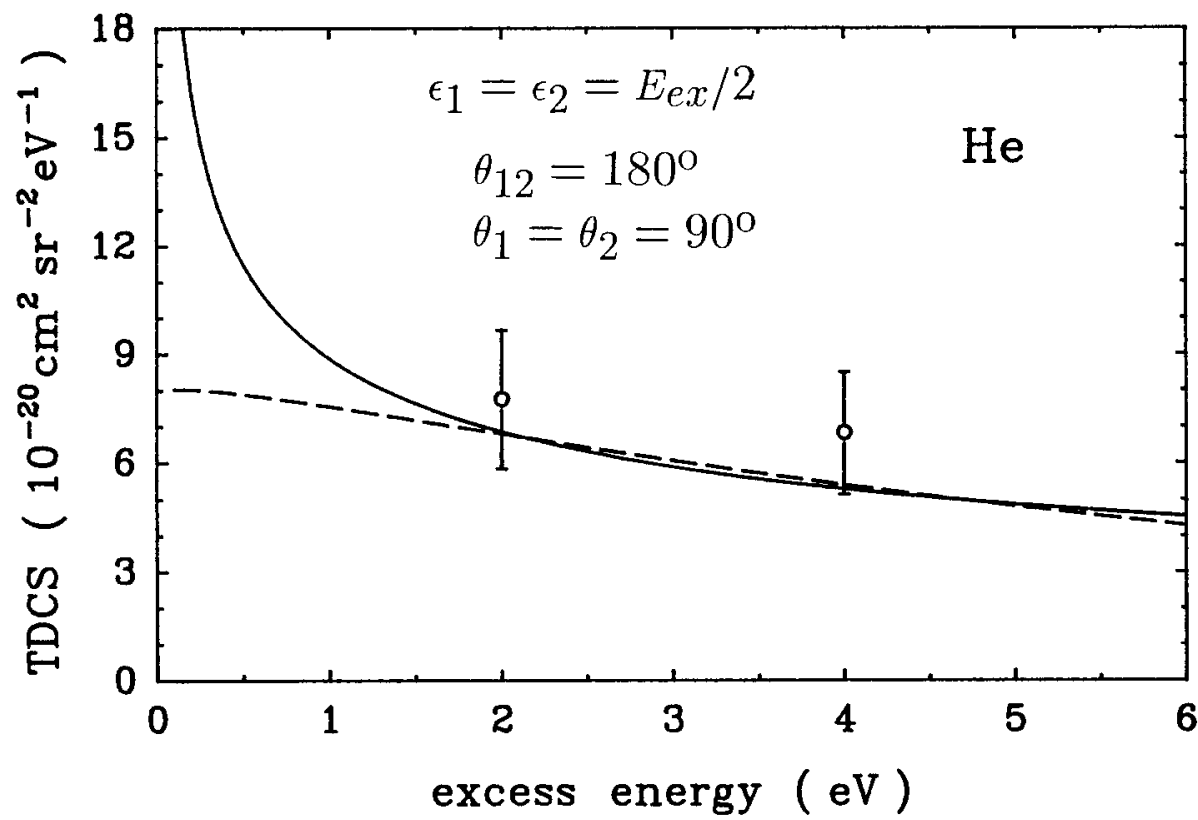

Figure 5. Triply differential (e,2e) cross sections for a He-atom target for final states having $\theta_{12}=\pi$ and $\theta_{1}=\theta_{2}=90^{\circ}$ and the continuum electrons sharing equally the excess energy in the range $0-6 \mathrm{cV}$. Open circles: experimental results of Rösel $e t$ al., Ref. [16]. Solid curve: theoretical results of Crothers, Ref. [11(a)]. Dashed curve: present first-order theoretical results. 
the calculations of Crothers [11(a)] and the effective charge calculations of Pan and Starace [2] and of Jones and Madison [17] at $\mathrm{E}_{\mathrm{x}}=2 \mathrm{eV}$ is not expected to hold for smaller excess energies. Figure 5 compares the results of our first-order calculations and the results of Crothers [11(a)] for the TDCS for He for the case of $\theta_{12}=\pi$ and $\theta_{1}=90^{\circ}$ in the energy range $0 \leq E_{x} \leq 6 \mathrm{eV}$. Crothers explicitly gives the TDCS results only for 1 and $2 \mathrm{eV}$ excess energies. Here we extract results for other energies using Fig. 1 and Eqs. (74) and (86) of Ref. [11(a)]; the TDCS as a function of the excess energy $E_{x}$ for the case of $\theta_{12}=\pi$ and $\theta_{1}=90^{\circ}$ is given by $8.9\left(\mathrm{E}_{\mathrm{x}} / \mathrm{eV}\right)^{-0.373} \times 10^{-20} \mathrm{~cm}^{2} \mathrm{sr}^{-2} \mathrm{eV}^{-1}$. The available absolute experimental results are also plotted [16]. One sees that although the two theoretical curves agree well with the available experimental results at and above $2 \mathrm{eV}$ excess energy, they depart from each other as the excess energy decreases below $2 \mathrm{eV}$. At present, the range of validity of the WPR theory is not known. Absolute experimental measurements in the region $\mathrm{E}_{\mathrm{x}}<2 \mathrm{eV}$ would provide an important test of the theory and of its range of validity.

\section{CONCLUSIONS}

We have presented in this paper distorted wave calculations for the TDCS for electron-impact ionization of $\mathrm{He}$ in the $\theta_{12}=\pi$ geometry. We find our results for equal energy sharing to be in good agreement with available absolute experimental measurements [16-18,21] over the range of excess energies $2 \mathrm{eV} \leq \mathrm{E}_{\mathrm{x}} \leq$ $80 \mathrm{eV}$. [Note, however, that our results do not include effects of doubly excited He resonance states and thus may not be reliable for $E_{\mathrm{x}} \geq 70 \mathrm{eV}$ in energy regions influenced by such resonances.]

For $E_{\mathrm{x}}=2 \mathrm{eV}$ we have found that inclusion of interchannel interactions between singlet and triplet partial waves is significant and is necessary to obtain good agreement with absolute experimental measurements $[16,17]$ for small and large angles (i.e., for $\theta_{1} \leq 50^{\circ}$ and $\theta_{1} \geq 130^{\circ}$ ). The polarization effects we have included were found to be negligible. However, for angles near the perpendicular plane (i.e., for $\theta_{1} \approx 90^{\circ}$ ) both interchannel interactions and ground-state correlations reduce the value of our first order TDCS's. Absolute experimental measurements for $\theta_{12}=\pi$ near the perpendicular plane thus may provide a sensitive test of such many-body correlation effects on the TDCS.

Finally, our neglect of Wannier-Peterkop-Rau (WPR) electron correlations near threshold is apparently not significant for $E_{x} \geq 2 \mathrm{eV}$. Over this region our first-order results agree very well with those of Crothers [11(a)], which include such WPR effects. As has been emphasized by Selles, Huetz, and Mazeau [5], and demonstrated in the comparison we present here of our results with those of Crothers for $\mathrm{E}_{\mathrm{X}}<2 \mathrm{eV}$, experimental measurements of the TDCS in this energy region may provide a significant test of WPR theory and of its range of validity.

\section{ACKNOWLEDGMENTS}

A. F. S. thanks F. H. M. Faisal and M. Ya. Amusia for helpful discussions. This work was supported in part by the U. S. National Science Foundation under Grant No. PHY-9108002.

\section{REFERENCES}

[1] Pan C. and Starace A. F., Phys. Rev. Lett. 67 (1991) 185-188.

[2] Pan C. and Starace A. F., Phys. Rev. A 45 (1992) 4588-4603.

[3] Pan C. and Starace A. F., Phys. Rev. A 47 (1993) 2389-2392.

[4] Pan C. and Starace A. F., in Many-Body Atomic Physics, edited by J. J. Boyle, A. Dalgarno, and M. S. Pindzola (Cambridge University Press, in press).

[5] Selles P., Huetz A., and Mazeau J., J. Phys. B 20 (1987) 5195-5212.

[6] Schlemmer P., Rösel T., Jung K., and Ehrhardt H., Phys. Rev. Lett. 63 (1989) 252-254. 
[7] Rösel T., Bär R., Jung K., and Ehrhardt H., Invited Papers and Progress Reports, European Conference on (e,2e) Collisions and Related Problems, edited by $\mathrm{H}$. Ehrhardt (Universität Kaiserslautern, Kaiserslautern, Germany, 1989) pp. 69-81.

[8] Selles P., Mazeau J., and Huetz A., J. Phys. B 23 (1990) 2613-2627.

[9] Brauner M., Briggs J. S., Klar H., Broad J. T., Rösel T., Jung K., and Ehrhardt H., J. Phys. B 24 (1991) 657-673.

[10] Jones S., Madison D. H., and Srivastava M. C., J. Phys. B 25 (1992) 1899-1914.

[11] (a) Crothers D. S. F., J. Phys. B 19 (1986) 463-483; (b) Carruthers D. R. J. and Crothers D. S. F., Z. Phys. D 23 (1992) 365-369.

[12] Peterkop R. K., Opt. Spektrosk. 13 (1962) 153-158, [Opt. Spectrosc. (USSR) 13 (1962) 87-89].

[13] Rudge M. R. H and Seaton M. J., Proc. R. Soc. London, Ser. A 283 (1965) 262-290.

[14] Jetzke S., Zaremba J., and Faisal F. H. M., Z. Phys. D 11 (1989) 63-69; Jetzke S. and Faisal F. H. M., J. Phys. B 25 (1992) 1543-1558.

[15] Cf. Table II of Ref. [2].

[16] Rösel T, Röder J., Frost L., Jung K., and Ehrhardt H., J. Phys. B 25 (1992) 3859-3872; Rösel T., Schlemmer P., Röder J., Frost L., Jung K., and Ehrhardt H., Z. Phys. D 23 (1992) 359-363.

[17] Rösel T., Röder J., Frost L., Jung K., Ehrhardt H., Jones S., and Madison D. H., Phys. Rev. A 46 (1992) 2539-2552.

[18] Murray A. J., Woolf M. B., and Read F. H., J. Phys B 25 (1992) 3021-3036.

[19] Wannier G. H., Phys. Rev. 90 (1953) 817-825.

[20] Peterkop R., (a) J. Phys. B 4 (1971) 513-521; (b) 16 (1983) L587-L593.

[21] Rau A. R. P., Phys. Rev. A 4 (1971) 207-220; Phys. Rep. 110 (1984) 369-387.

[22] Gélébart F. and Tweed R. J., J. Phys. B 23 (1990) L641-L647.

[23] Pochat A., Zhang X., Whelan C. T., Walters H. R. J., Tweed R. J., Gélébart F., Cherid M., and Allan R. J., Phys. Rev. 47 (1993) R3483-R3486. 